\title{
Existence of positive solutions for two-point boundary value problems of nonlinear fractional $q$-difference equation
}

\author{
Caixia Guo*, Jianmin Guo, Ying Gao and Shugui Kang
}

"Correspondence: iris-gcx@163.com School of Mathematics and Computer Sciences, Shanxi Datong University, Datong, People's Republic of China

\section{严 Springer}

\begin{abstract}
This paper is concerned with the two-point boundary value problems of a nonlinear fractional $q$-difference equation with dependence on the first order $q$-derivative. We discuss some new properties of the Green function by using $q$-difference calculus. Furthermore, by means of Schauder's fixed point theorem and an extension of Krasnoselskii's fixed point theorem in a cone, the existence of one positive solution and of at least one positive solution for the boundary value problem is established.
\end{abstract}

MSC: $34 \mathrm{~A} 08 ; 34 \mathrm{~K} 30$

Keywords: Fractional q-difference calculus; Two-point boundary value problem; Fixed point theorem; Positive solution

\section{Introduction}

The term " $q$-difference" actually refers to quantum difference. Quantum calculus, sometimes called calculus without limits, is equivalent to traditional infinitesimal calculus without the notion of limits. It defines " $q$-calculus" and " $h$-calculus", where $h$ ostensibly stands for Planck's constant while $q$ stands for quantum. The two parameters are related by the formula $q=e^{i a}=e^{2 \pi i h}$, where $h=\frac{a}{2 \pi}$ is the reduced Planck constant. The $q$-calculus, dating in a sense back to Euler and Jacobi [1-3], is only recently beginning to see more usefulness and a lot of applications in quantum mechanics, having an intimate connection with commutativity relations. Based on this, there have been published a lot of papers about fractional $q$-calculus and fractional $q$-differential equation theory. At the same time, the topic of the fractional quantum difference equation has also attracted the attention of many researchers in recent years (see [4-6] and the references therein). In recent years, some boundary value problems with fractional $q$-differences have aroused heated discussion among many authors [7-22]. They obtained many results as regards the existence and multiplicity of nontrivial solutions, positive solutions, negative solutions and extremal solutions by applying some well-known tools of fixed point theory such as the Banach contraction principle, the Guo-Krasnosel'skii fixed point theorem on cones, monotone iterative methods and Leray-Schauder degree theory.

But we find that the discussed nonlinear terms is only $f(t, u(t))$ in the literature above (see, e.g., [7]), and there is little literature treating nonlinear terms with a first order

(c) The Author(s) 2018. This article is distributed under the terms of the Creative Commons Attribution 4.0 International License (http://creativecommons.org/licenses/by/4.0/), which permits unrestricted use, distribution, and reproduction in any medium, provided you give appropriate credit to the original author(s) and the source, provide a link to the Creative Commons license, and indicate if changes were made. 
$q$-derivative, which is our focus in the study. Based on this, in this paper, we will be interested in the $q$-analog of the fractional differential problem given by

$$
\left\{\begin{array}{l}
\left(D_{q}^{\alpha} y\right)(t)=-f\left(t, y(t), D_{q} y(t)\right), \quad 0<t<1, \\
y(0)=\left(D_{q} y\right)(0)=\left(D_{q} y\right)(1)=0,
\end{array}\right.
$$

where $2<\alpha \leq 3, f:[0,1] \times[0,+\infty) \times \mathbb{R} \rightarrow[0,+\infty)$ is continuous. In Sect. 2 , we will give some necessary definitions of fractional $q$-calculus and deduce the new properties of the Green function. In Sect. 3, by means of Schauder's fixed point theorem and Krasnoselskii fixed point theorem in a cone, some results on the existence of positive solutions of problem (1.1) are established. Finally, an example is given to illustrate the main results of this paper.

\section{Preliminaries}

For convenience, we collect here the necessary definitions from the theory of fractional $q$-calculus.

Let $q \in(0,1)$ and define

$$
[a]_{q}=\frac{1-q^{a}}{1-q}, \quad a \in \mathbb{R} .
$$

The $q$-analog of the power function $(a-b)^{n}$ with $n \in \mathbb{N}_{0}$ is

$$
(a-b)^{0}=1, \quad(a-b)^{n}=\prod_{k=0}^{n-1}\left(a-b q^{k}\right), \quad n \in \mathbb{N}, a, b \in \mathbb{R} .
$$

More generally, if $\alpha \in \mathbb{R}$, then

$$
(a-b)^{(\alpha)}=a^{\alpha} \prod_{n=0}^{\infty} \frac{a-b q^{n}}{a-b q^{\alpha+n}} .
$$

If $b=0$, then $(a-b)^{(\alpha)}=a^{(\alpha)}=a^{\alpha}$. It is easy to see that $[a(t-s)]^{(\alpha)}=a^{\alpha}(t-s)^{(\alpha)}$ and $(a-$ $b)^{(\alpha)}=\left(a-b q^{\alpha-1}\right)(a-b)^{(\alpha-1)}$. There are two important results:

(1) If $\alpha>0, a \leq b \leq t$, then $(t-a)^{(\alpha)} \geq(t-b)^{(\alpha)}$.

(2) If $\alpha<0, a \leq b \leq t$, then $(t-a)^{(\alpha)} \leq(t-b)^{(\alpha)}$.

Result (1) comes from the Remark 2.1 of [8]. For result (2), since $(t-a)^{(\alpha)}=t^{\alpha} \prod_{n=0}^{\infty} \frac{t-a q^{n}}{t-a q^{\alpha+n}}$ and $(t-b)^{(\alpha)}=t^{\alpha} \prod_{n=0}^{\infty} \frac{t-b q^{n}}{t-b q^{\alpha+n}}$, it is sufficient to show that

$$
\begin{aligned}
\left(t-a q^{n}\right)\left(t-b q^{\alpha+n}\right) \leq\left(t-b q^{n}\right)\left(t-a q^{\alpha+n}\right) \\
\quad \Leftrightarrow \quad t^{2}-t b q^{\alpha+n}-t a q^{n}+a b q^{\alpha+2 n} \leq t^{2}-t a q^{\alpha+n}-t a q^{n}+a b q^{\alpha+2 n} \\
\Leftrightarrow \quad q^{n}\left(a+b q^{\alpha}\right) \geq q^{n}\left(b+a q^{\alpha}\right) \\
\Leftrightarrow \quad a+b q^{\alpha} \geq b+a q^{\alpha} \\
\Leftrightarrow \quad(b-a) q^{\alpha} \geq b-a \\
\Leftrightarrow \quad q^{\alpha} \geq 1 .
\end{aligned}
$$


The final expression is obtained by $\alpha<0,0<q<1$. This completes the proof of the result (2).

The $q$-gamma function is defined by

$$
\Gamma_{q}(x)=\frac{(1-q)^{(x-1)}}{(1-q)^{x-1}}, \quad x \in \mathbb{R} \backslash\{0,-1,-2, \ldots\},
$$

and satisfies $\Gamma_{q}(x+1)=[x]_{q} \Gamma_{q}(x)$.

The expression

$$
D_{q} f(x)=\frac{f(q x)-f(x)}{(q-1) x}
$$

is called the $q$-derivative of the function $f(x)$. $D_{q}$ has the following properties:

$$
\begin{aligned}
& D_{q}(a f(x)+b g(x))=a D_{q} f(x)+b D_{q} g(x), \\
& D_{q}(f(x) g(x))=f(x) D_{q} g(x)+g(q x) D_{q} f(x) ; \\
& { }_{t} D_{q}(t-s)^{(\alpha)}=[\alpha]_{q}(t-s)^{(\alpha-1)}, \\
& \left({ }_{x} D_{q} \int_{0}^{x} f(x, t) d_{q} t\right)(x)=\int_{0}^{x} D_{x} f(x, t) d_{q} t+f(q x, x) .
\end{aligned}
$$

The $q$-integral of a function $f$ defined on the interval $[0, b]$ is given by

$$
I_{q} f(x)=\int_{0}^{x} f(t) d_{q} t=x(1-q) \sum_{n=0}^{\infty} f\left(x q^{n}\right) q^{n}, \quad x \in[0, b] .
$$

If $a \in[0, b]$ and $f$ is defined on the interval $[0, b]$, its integral from $a$ to $b$ is defined by

$$
\int_{a}^{b} f(t) d_{q} t=\int_{0}^{b} f(t) d_{q} t-\int_{0}^{a} f(t) d_{q} t
$$

Basic properties of the two operators can be found in [23]:

$$
D_{q} I_{q} f(x)=f(x),
$$

and if $f$ is continuous at $x=0$, then

$$
I_{q} D_{q} f(x)=f(x)-f(0) .
$$

Definition 2.1 ([24]) Let $\alpha \geq 0$ and $f$ be a function defined on [0,1]. The fractional $q$ integral of the Riemann-Liouville type is $\left(I_{q}^{0} f\right)(x)=f(x)$ and

$$
\left(I_{q}^{\alpha} f\right)(x)=\frac{1}{\Gamma_{q}(\alpha)} \int_{0}^{x}(x-q t)^{(\alpha-1)} f(t) d_{q} t, \quad \alpha>0, x \in[0,1] .
$$

Definition 2.2 ([25]) The fractional $q$-derivative of the Riemann-Liouville type of order $\alpha \geq 0$ is defined by

$$
\left(D_{q}^{\alpha} f\right)(x)=\left(D_{q}^{m} I_{q}^{m-\alpha} f\right)(x), \quad \alpha>0,
$$

where $m$ is the smallest integer greater than or equal to $\alpha$. 
Lemma 2.1 ([24]) Let $\alpha, \gamma \geq 0$ and $f$ be a function defined on [0,1]. Then the next formulas hold:

$$
\begin{aligned}
& \left(I_{q}^{\beta} I_{q}^{\alpha} f\right)(x)=\left(I_{q}^{\alpha+\beta} f\right)(x), \\
& \left(D_{q}^{\alpha} I_{q}^{\alpha} f\right)(x)=f(x) .
\end{aligned}
$$

Definition 2.3 ([26]) Let $\alpha>0$ and $p$ be a positive integer. Then the following equality holds:

$$
\left(I_{q}^{\alpha} D_{q}^{p} f\right)(x)=\left(D_{q}^{p} I_{q}^{\alpha} f\right)(x)-\sum_{k=0}^{p} \frac{x^{\alpha-p+k}}{\Gamma_{q}(\alpha+k-p+1)}\left(D_{q}^{k} f\right)(0) .
$$

Lemma 2.2 ([7]) The unique solution of the q-analog of the fractional differential problem (1.1) is given by

$$
y(t):=\int_{0}^{1} G(t, q s) f\left(s, y(s), D_{q} y(s)\right) d_{q} s
$$

where $2<\alpha \leq 3, G(t, q s)$ is the Green function for the problem (1.1), which is given by

$$
G(t, q s)=\frac{1}{\Gamma_{q}(\alpha)} \begin{cases}t^{\alpha-1}(1-q s)^{(\alpha-2)}-(t-q s)^{(\alpha-1)}, & 0 \leq q s \leq t \leq 1 \\ t^{\alpha-1}(1-q s)^{(\alpha-2)}, & 0 \leq t \leq q s \leq 1\end{cases}
$$

Lemma 2.3 The Green function $G(t, q s)$ defined as in the statement of Lemma 2.2 satisfies the following conditions:

(1) $G(t, q s)>0$ and $G(t, q s) \leq G(1, q s)$ for each $(t, s) \in[0,1] \times[0,1]$;

(2) $G(t, q s) \geq g(t) G(1, q s)$ for each $(t, s) \in[0,1] \times[0,1]$ with $g(t)=t^{\alpha-1}$;

(3) For $s \in[0,1], \min _{t \in\left[\frac{1}{4}, \frac{3}{4}\right]} G(t, q s) \geq\left(\frac{1}{4}\right)^{\alpha-1} G(1, q s)$.

Proof Proofs of (1) and (2) are given in [7].

(3) Let $g_{1}(t, q s)=t^{\alpha-1}(1-q s)^{(\alpha-2)}-(t-q s)^{(\alpha-1)}, 0 \leq q s \leq t \leq 1, g_{2}(t, q s)=t^{\alpha-1}(1-q s)^{(\alpha-2)}$, $0 \leq t \leq q s \leq 1$. For $0 \leq q s \leq t \leq 1$,

$$
\begin{aligned}
{ }_{t} D_{q} g_{1}(t, q s) & =[\alpha-1]_{q} t^{\alpha-2}(1-q s)^{(\alpha-2)}-[\alpha-1]_{q}(t-q s)^{(\alpha-2)} \\
& \geq[\alpha-1]_{q} t^{\alpha-2}(1-q s)^{(\alpha-2)}-[\alpha-1]_{q}(t-t q s)^{(\alpha-2)} \\
& =[\alpha-1]_{q} t^{\alpha-2}(1-q s)^{(\alpha-2)}-[\alpha-1]_{q} t^{\alpha-2}(1-q s)^{(\alpha-2)} \\
& =0
\end{aligned}
$$

so $g_{1}(t, q s)$ is increasing with respect to $t$. We have

$$
\begin{aligned}
\min _{\frac{1}{4} \leq t \leq \frac{3}{4}} g_{1}(t, q s) & \geq \frac{1}{\Gamma_{q}(\alpha)}\left[(1-q s)^{(\alpha-2)}\left(\frac{1}{4}\right)^{\alpha-1}-\left(\frac{1}{4}-q s\right)^{(\alpha-1)}\right] \\
& \geq \frac{1}{\Gamma_{q}(\alpha)}\left[(1-q s)^{(\alpha-2)}\left(\frac{1}{4}\right)^{\alpha-1}-\left(\frac{1}{4}-\frac{1}{4} q s\right)^{(\alpha-1)}\right]
\end{aligned}
$$




$$
\begin{aligned}
& =\frac{1}{\Gamma_{q}(\alpha) 4^{\alpha-1}}\left[(1-q s)^{(\alpha-2)}-(1-q s)^{(\alpha-1)}\right] \\
& =\frac{1}{\Gamma_{q}(\alpha) 4^{\alpha-1}}\left[(1-q s)^{(\alpha-2)}-\left(1-q s q^{\alpha-2}\right)(1-q s)^{(\alpha-2)}\right] \\
& =\frac{1}{\Gamma_{q}(\alpha) 4^{\alpha-1}}(1-q s)^{(\alpha-2)} s q^{\alpha-1} \\
& =\left(\frac{1}{4}\right)^{\alpha-1} G(1, q s) .
\end{aligned}
$$

It is easy to see that $g_{2}(t, q s)$ is increasing with respect to $t$. We have

$$
\min _{\frac{1}{4} \leq t \leq \frac{3}{4}} g_{2}(t, q s) \geq \frac{1}{\Gamma_{q}(\alpha)}(1-q s)^{(\alpha-2)}\left(\frac{1}{4}\right)^{\alpha-1} \geq\left(\frac{1}{4}\right)^{\alpha-1} G(1, q s) .
$$

Obviously, $\min _{\frac{1}{4} \leq t \leq \frac{3}{4}} G(t, q s) \geq\left(\frac{1}{4}\right)^{\alpha-1} G(1, q s)$.

Definition 2.4 Let $E$ be a real Banach space. $A$ nonempty convex closed set $P$ is called a cone provided that: (1) $a u \in P$, for all $u \in P ; a \geq 0$; (2) $u,-u \in P$ implies $u=0$.

Let $X$ be a Banach space and $P \subset X$ a cone. Suppose $\alpha, \beta: X \rightarrow \mathbb{R}^{+}$are two continuous convex functions satisfying

$$
\alpha(\lambda u)=|\lambda| \alpha(u), \quad \beta(\lambda u)=|\lambda| \beta(u),
$$

for $u \in X, \lambda \in \mathbb{R}$, and

$$
\|u\| \leq \kappa \max \{\alpha(u), \beta(u)\}
$$

for $u \in X$, and $\alpha\left(u_{1}\right) \geq \alpha\left(u_{2}\right)$ for $u_{1}, u_{2} \in P, u_{1} \leq u_{2}$, where $\kappa>0$ is a constant.

Lemma 2.4 ([27]) Let $r_{2}>r_{1}>0, L>0$ be constants and $\Omega_{i}=\left\{u \in X: \alpha(u)<r_{i}, \beta(u)<\right.$ $L\}, i=1,2$ be two bounded open sets in $X$. Set $D_{i}=\left\{u \in X: \alpha(u)=r_{i}\right\}$. Assume $T: P \rightarrow P$ is a completely continuous operator satisfying

$\left(C_{1}\right) \alpha(T u)<r_{1}, u \in D_{1} \cap P ; \alpha(T u)>r_{2}, u \in D_{2} \cap P$;

$\left(C_{2}\right) \beta(T u)<L, u \in P$;

$\left(C_{3}\right)$ there is a $p \in\left(\Omega_{2} \cap P\right) \backslash\{0\}$ such that $\alpha(p) \neq 0$ and $\alpha(u+\lambda p) \geq \alpha(u)$ for all $u \in P$ and $\lambda \geq 0$.

Then $T$ has at least one fixed point in $\left(\Omega_{2} \backslash \bar{\Omega}_{1}\right) \cap P$.

\section{Main results and proofs}

Consider the Banach space

$$
X=\left\{y: y(t) \in C[0,1] \cap C^{1}[0,1]\right\}
$$

with the norm

$$
\|y\|=\left[\|y\|_{0}^{2}+\left\|D_{q} y\right\|_{0}^{2}\right]^{\frac{1}{2}}
$$


where $\|y\|_{0}=\max _{0 \leq t \leq 1}|y(t)|,\left\|D_{q} y\right\|_{0}=\max _{0 \leq t \leq 1}\left|D_{q} y(t)\right|$. Define the cone on $X: P=\{y \in$ $X: y(t) \geq 0\}$ and functionals $\alpha(y)=\max _{0 \leq t \leq 1}|y(t)|, \beta(y)=\max _{0 \leq t \leq 1}\left|D_{q} y(t)\right|$, then we obtain $\alpha(\lambda y)=|\lambda| \alpha(y), \beta(\lambda y)=|\lambda| \beta(y)$, for $y \in X, \lambda \in \mathbb{R}, \alpha\left(y_{1}\right) \geq \alpha\left(y_{2}\right)$ for $y_{1}, y_{2} \in P, y_{1} \leq y_{2}$. For all $y(t) \in C[0,1] \cap C^{1}[0,1]$, define

$$
T y(t):=\int_{0}^{1} G(t, q s) f\left(s, y(s), D_{q} y(s)\right) d_{q} s
$$

For convenience, we introduce the following notations:

$$
\begin{aligned}
N= & \int_{0}^{1} G(1, q s) d_{q} s, \quad Q=\int_{0}^{1} G(1, q s) a(s) d_{q} s, \\
R= & \frac{1}{16} \int_{\frac{1}{4}}^{\frac{3}{4}} G(1, q s) d_{q} s, \quad W=2 \int_{0}^{1} \frac{(1-q s)^{(\alpha-2)}}{\Gamma_{q}(\alpha-1)} d_{q} s, \\
\tau= & \max _{0 \leq t \leq 1}\left(\int_{0}^{t} \frac{(t-q s)^{(\alpha-2)}}{\Gamma_{q}(\alpha-1)} d_{q} s+\int_{0}^{t} \frac{(t-q s)^{(\alpha-2)}}{\Gamma_{q}(\alpha-1)} d_{q} s\right)+\int_{0}^{1} \frac{(1-q s)^{(\alpha-2)} t^{\alpha-2}}{\Gamma_{q}(\alpha-1)} a(s) d_{q} s \\
& +\int_{0}^{1} \frac{(1-q s)^{(\alpha-2)} t^{\alpha-2}}{\Gamma_{q}(\alpha-1)} a(s) d_{q} s .
\end{aligned}
$$

In this section, we will give the existence results of a positive solution to the boundary value problem (1.1) on the basis of Lemma 3.1 and make the following assumptions:

$\left(\mathrm{H}_{0}\right)$ There exists a nonnegative function $a(t) \in L(0,1) \cap C[0,1]$ such that

$$
|f(t, u, v)| \leq a(t)+\kappa_{1}|u|^{\sigma_{1}}+\kappa_{2}|v|^{\sigma_{2}}, \quad \kappa_{i}>0,0<\sigma_{i}<1, i=1,2
$$

We also suppose that there exist $L>b>\frac{1}{16} b>c>0$ such that $f(t, u, v)$ satisfies the following conditions:

$\left(\mathrm{H}_{1}\right) f(t, u, v)<c / N$ for $(t, u, v) \in[0,1] \times[0, c] \times[-L, L]$;

$\left(\mathrm{H}_{2}\right) f(t, u, v) \geq b / R$ for $(t, u, v) \in[0,1] \times\left[\frac{1}{16} b, b\right] \times[-L, L]$;

$\left(\mathrm{H}_{3}\right) f(t, u, v)<L / W$ for $(t, u, v) \in[0,1] \times[0, b] \times[-L, L]$.

Lemma 3.1 Suppose that $f\left(t, y, D_{q} y\right)$ is continuous on $[0,1] \times[0,+\infty) \times \mathbb{R}$. Then the mapping $T: P \rightarrow P$ is completely continuous.

Proof In view of the expression of $G(t, q s)$, it is clear that $T y \in C[0,1] \cap C^{1}[0,1], T y(t) \geq 0$, and $T y(t)$ is continuous. Hence $T: P \rightarrow P$.

Next, we show that $T$ is uniformly bounded. Let $D \subset P$ be bounded, i.e. there exists a real number $L>0$ such that $\|y\| \leq L$, for all $y \in D$. Let $M=\max _{0 \leq t \leq 1,0 \leq y \leq L, 0 \leq D_{q} y \leq L} \mid f(t, y$, $\left.D_{q} y\right) \mid+1$, then, for $y \in D$, from Lemma 2.3, on the one hand, one has

$$
\begin{aligned}
|T y(t)| & \leq \int_{0}^{1}\left|G(t, q s) f\left(s, y(s), D_{q} y(s)\right)\right| d_{q} s \\
& \leq M \int_{0}^{1} G(1, q s) d_{q} s \\
& \leq 2 M \int_{0}^{1} \frac{(1-q s)^{(\alpha-2)}}{\Gamma_{q}(\alpha-1)} d_{q} s .
\end{aligned}
$$


On the other hand,

$$
\begin{aligned}
\left|{ }_{t} D_{q}(T y)(t)\right|= & \mid \int_{0}^{1} \frac{(1-q s)^{(\alpha-2)} t^{\alpha-2}}{\Gamma_{q}(\alpha-1)} f\left(s, y(s), D_{q} y(s)\right) d_{q} s \\
& -\int_{0}^{t} \frac{(t-q s)^{(\alpha-2)}}{\Gamma_{q}(\alpha-1)} f\left(s, y(s), D_{q} y(s)\right) d_{q} s \mid \\
\leq & M\left[\int_{0}^{1} \frac{(1-q s)^{(\alpha-2)} t^{\alpha-2}}{\Gamma_{q}(\alpha-1)} d_{q} s+\int_{0}^{t} \frac{(t-q s)^{(\alpha-2)}}{\Gamma_{q}(\alpha-1)} d_{q} s\right] \\
\leq & 2 M \int_{0}^{1} \frac{(1-q s)^{(\alpha-2)}}{\Gamma_{q}(\alpha-1)} d_{q} s .
\end{aligned}
$$

Then we see that $T(D)$ is bounded.

Finally, we show that $T$ is equi-continuous. For all $\varepsilon>0, \exists \delta=\min \left\{\frac{1}{2}, \frac{\varepsilon \Gamma(\alpha-1)}{2 M}\right\}>0$, let $t_{1}, t_{2} \in[0,1], t_{1}<t_{2}, y \in D$, for $0<t_{2}-t_{1}<\delta$, we have

$$
\begin{aligned}
\left|T y\left(t_{2}\right)-T y\left(t_{1}\right)\right| \leq & \int_{0}^{1}\left|G\left(t_{2}, q s\right)-G\left(t_{1}, q s\right)\right| f\left(s, y(s), D_{q} y(s)\right) \mid d_{q} s \\
\leq & M\left[\int_{0}^{t_{1}} \frac{(1-q s)^{(\alpha-2)}\left(t_{2}^{\alpha-1}-t_{1}^{\alpha-1}\right)+\left(t_{1}-q s\right)^{(\alpha-1)}-\left(t_{2}-q s\right)^{(\alpha-1)}}{\Gamma_{q}(\alpha)} d_{q} s\right. \\
& +\int_{t_{1}}^{t_{2}} \frac{(1-q s)^{(\alpha-2)}\left(t_{2}^{\alpha-1}-t_{1}^{\alpha-1}\right)-\left(t_{2}-q s\right)^{(\alpha-1)}}{\Gamma_{q}(\alpha)} d_{q} s \\
& \left.+\int_{t_{2}}^{1} \frac{(1-q s)^{(\alpha-2)}\left(t_{1}^{\alpha-1}-t_{2}^{\alpha-1}\right)}{\Gamma_{q}(\alpha)} d_{q} s\right] \\
\leq & M\left[\frac{t_{2}^{\alpha-1}-t_{1}^{\alpha-1}}{\Gamma_{q}(\alpha)} \int_{0}^{1}(1-q s)^{(\alpha-2)} d_{q} s-\int_{0}^{t_{2}} \frac{\left(t_{2}-q s\right)^{(\alpha-1)}}{\Gamma_{q}(\alpha)} d_{q} s\right. \\
& \left.+\int_{0}^{t_{1}} \frac{\left(t_{1}-q s\right)^{(\alpha-1)}}{\Gamma_{q}(\alpha)} d_{q} s\right] .
\end{aligned}
$$

Let $\varphi(t)=\int_{0}^{t}(t-q s)^{(\alpha-1)} d_{q} s$, since ${ }_{t} D_{q} \varphi(t)=\int_{0}^{t}[\alpha-1]_{q}(t-q s)^{(\alpha-2)} d_{q} s \geq 0$, we have $\varphi\left(t_{1}\right) \leq$ $\varphi\left(t_{2}\right)$. So we have

$$
\left|T y\left(t_{2}\right)-T y\left(t_{1}\right)\right| \leq \frac{M}{\Gamma_{q}(\alpha)}\left(t_{2}^{\alpha-1}-t_{1}^{\alpha-1}\right) .
$$

Since

$$
\begin{aligned}
{ }_{t} D_{q}\left({ }_{t} D_{q}(T y(t))\right) \\
={ }_{t} D_{q}\left(\int_{0}^{1} \frac{(1-q s)^{(\alpha-2)} t^{\alpha-2}}{\Gamma_{q}(\alpha-1)} f\left(s, y(s), D_{q} y(s)\right) d_{q} s\right. \\
\left.\quad-\int_{0}^{t} \frac{(t-q s)^{(\alpha-2)}}{\Gamma_{q}(\alpha-1)} f\left(s, y(s), D_{q} y(s)\right) d_{q} s\right) \\
=\int_{0}^{1} \frac{(1-q s)^{(\alpha-2)} t^{\alpha-3}}{\Gamma_{q}(\alpha-2)} f\left(s, y(s), D_{q} y(s)\right) d_{q} s-\int_{0}^{t} \frac{(t-q s)^{(\alpha-3)}}{\Gamma_{q}(\alpha-2)} f\left(s, y(s), D_{q} y(s)\right) d_{q} s \\
\leq \int_{0}^{1} \frac{(1-q s)^{(\alpha-2)} t^{\alpha-3}}{\Gamma_{q}(\alpha-2)} f\left(s, y(s), D_{q} y(s)\right) d_{q} s-\int_{0}^{t} \frac{(t-t q s)^{(\alpha-3)}}{\Gamma_{q}(\alpha-2)} f\left(s, y(s), D_{q} y(s)\right) d_{q} s \\
\leq 0
\end{aligned}
$$


we have

$$
\begin{aligned}
\left|{ }_{t} D_{q}(T y)\left(t_{2}\right)-{ }_{t} D_{q}(T y)\left(t_{1}\right)\right| & \int_{0}^{1} \frac{(1-q s)^{(\alpha-2)} t_{1}^{\alpha-2}}{\Gamma_{q}(\alpha-1)} f\left(s, y(s), D_{q} y(s)\right) d_{q} s-\int_{0}^{t_{1}} \frac{\left(t_{1}-q s\right)^{(\alpha-2)}}{\Gamma_{q}(\alpha-1)} f\left(s, y(s), D_{q} y(s)\right) d_{q} s \\
& -\int_{0}^{1} \frac{(1-q s)^{(\alpha-2)} t_{2}^{\alpha-2}}{\Gamma_{q}(\alpha-1)} f\left(s, y(s), D_{q} y(s)\right) d_{q} s \\
& +\int_{0}^{t_{2}} \frac{\left(t_{2}-q s\right)^{(\alpha-2)}}{\Gamma_{q}(\alpha-1)} f\left(s, y(s), D_{q} y(s)\right) d_{q} s \mid \\
\leq & M\left[\frac{t_{1}^{\alpha-2}-t_{2}^{\alpha-2}}{\Gamma_{q}(\alpha-1)} \int_{0}^{1}(1-q s)^{(\alpha-2)} d_{q} s-\int_{0}^{t_{1}} \frac{\left(t_{1}-q s\right)^{(\alpha-2)}}{\Gamma_{q}(\alpha-1)} d_{q} s\right. \\
& \left.+\int_{0}^{t_{2}} \frac{\left(t_{2}-q s\right)^{(\alpha-2)}}{\Gamma_{q}(\alpha-1)} d_{q} s\right] \\
= & \frac{M}{\Gamma_{q}(\alpha-1)}\left[\left(t_{1}^{\alpha-2}-t_{2}^{\alpha-2}\right)(1-q) \sum_{n=0}^{\infty}\left(1-q^{n+1}\right)^{(\alpha-2)} q^{n}\right. \\
& \left.-t_{1}(1-q) \sum_{n=0}^{\infty}\left(t_{1}-t_{1} q^{n+1}\right)^{(\alpha-2)} q^{n}+t_{2}(1-q) \sum_{n=0}^{\infty}\left(t_{2}-t_{2} q^{n+1}\right)^{(\alpha-2)} q^{n}\right] \\
= & \frac{M}{\Gamma_{q}(\alpha-1)}\left[\left(t_{2}^{(\alpha-1)}-t_{1}^{(\alpha-1)}\right)-\left(t_{2}^{(\alpha-2)}-t_{1}^{(\alpha-2)}\right)\right](1-q) \sum_{n=0}^{\infty}\left(1-q^{n+1}\right)^{(\alpha-2)} q^{n} \\
\leq & \frac{M}{\Gamma_{q}(\alpha-1)}\left[\left(t_{2}^{(\alpha-1)}-t_{1}^{(\alpha-1)}\right)-\left(t_{2}^{(\alpha-2)}-t_{1}^{(\alpha-2)}\right)\right] \\
\leq & \frac{M}{\Gamma_{q}(\alpha-1)}\left(t_{2}^{(\alpha-1)}-t_{1}^{(\alpha-1)}\right) .
\end{aligned}
$$

Case 1: for $0 \leq t_{1}<\delta, \delta \leq t_{2}<2 \delta, t_{2}^{\alpha-1}-t_{1}^{\alpha-1} \leq t_{2}^{\alpha-1}<(2 \delta)^{\alpha-1}<2 \delta$.

Case 2: for $0 \leq t_{1}<t_{2} \leq \delta, t_{2}^{\alpha-1}-t_{1}^{\alpha-1} \leq t_{2}^{\alpha-1}<\delta^{\alpha-1}=\delta \cdot \delta^{\alpha-2}<2 \delta$.

Case 3: for $\delta \leq t_{1}<t_{2} \leq 1$, by means of differential mean value theorem, we get $t_{2}^{\alpha-1}-$ $t_{1}^{\alpha-1} \leq(\alpha-1)\left(t_{2}-t_{1}\right) \leq 2 \delta$.

Hence, we obtain

$$
\begin{aligned}
& \left|T y\left(t_{2}\right)-T y\left(t_{1}\right)\right|<\frac{2 M \delta}{\Gamma_{q}(\alpha)}<\varepsilon, \\
& \left|{ }_{t} D_{q}(T y)\left(t_{2}\right)-{ }_{t} D_{q}(T y)\left(t_{1}\right)\right|<\frac{2 M \delta}{\Gamma_{q}(\alpha-1)}<\varepsilon .
\end{aligned}
$$

In view of the Arzela-Ascoli theorem, it is easy to see that $T: P \rightarrow P$ is completely continuous.

Theorem 3.1 Suppose $f:[0,1] \times[0,+\infty) \times \mathbb{R} \rightarrow[0,+\infty)$ is continuous, and $f$ satisfies $\left(\mathrm{H}_{0}\right)$. Then problem (1.1) has one positive solution. 
Proof Let $\overline{P_{a}}=\{u: u \in P,\|u\| \leq a\}$, where $a \geq \max \left\{\left(8 \kappa_{1} \tau\right)^{\frac{1}{1-\sigma_{1}}},\left(8 \kappa_{1} N\right)^{\frac{1}{1-\sigma_{1}}},\left(8 \kappa_{2} \tau\right)^{\frac{1}{1-\sigma_{2}}}\right.$, $\left.\left(8 \kappa_{2} N\right)^{\frac{1}{1-\sigma_{2}}}, 4 \tau, 4 Q\right\}$. In the following, we show that $T: \overline{P_{a}} \rightarrow \overline{P_{a}}$. If $y \in \overline{P_{a}}$, it follows that

$$
\begin{aligned}
& 0 \leq y(t) \leq \max _{0 \leq t \leq 1}|y(t)| \leq\|y\| \leq a, \\
& 0 \leq D_{q} y(t) \leq \max _{0 \leq t \leq 1}\left|D_{q} y(t)\right| \leq\|y\| \leq a .
\end{aligned}
$$

Thus

$$
\left|f\left(t, y, D_{q} y\right)\right| \leq a(t)+\kappa_{1}|a|^{\sigma_{1}}+\kappa_{2}|a|^{\sigma_{2}}, \quad \kappa_{i}>0,0<\sigma_{i}<1, i=1,2
$$

Further, we have

$$
\begin{aligned}
|T y(t)| & \leq \int_{0}^{1}\left|G(t, q s) f\left(s, y(s), D_{q} y(s)\right)\right| d_{q} s \\
& \leq\left(\kappa_{1}|a|^{\sigma_{1}}+\kappa_{2}|a|^{\sigma_{2}}\right) \int_{0}^{1} G(1, q s) d_{q} s+\int_{0}^{1} G(1, q s) a(s) d_{q} s
\end{aligned}
$$

and

$$
\begin{aligned}
\left|{ }_{t} D_{q}(T y)(t)\right|= & \mid \int_{0}^{1} \frac{(1-q s)^{(\alpha-2)} t^{\alpha-2}}{\Gamma_{q}(\alpha-1)} f\left(s, y(s), D_{q} y(s)\right) d_{q} s \\
& -\int_{0}^{t} \frac{(t-q s)^{(\alpha-2)}}{\Gamma_{q}(\alpha-1)} f\left(s, y(s), D_{q} y(s)\right) d_{q} s \mid \\
\leq & \left(\kappa_{1}|a|^{\sigma_{1}}+\kappa_{2}|a|^{\sigma_{2}}\right)\left(\int_{0}^{1} \frac{(1-q s)^{(\alpha-2)} t^{\alpha-2}}{\Gamma_{q}(\alpha-1)} d_{q} s+\int_{0}^{t} \frac{(t-q s)^{(\alpha-2)}}{\Gamma_{q}(\alpha-1)} d_{q} s\right) \\
& +\int_{0}^{1} \frac{(1-q s)^{(\alpha-2)} t^{\alpha-2}}{\Gamma_{q}(\alpha-1)} a(s) d_{q} s+\int_{0}^{t} \frac{(t-q s)^{(\alpha-2)}}{\Gamma_{q}(\alpha-1)} a(s) d_{q} s .
\end{aligned}
$$

Furthermore,

$$
\begin{aligned}
\|T y\| & =\left[\|T y\|_{0}^{2}+\left\|D_{q}(T y)\right\|_{0}^{2}\right]^{\frac{1}{2}} \leq \max _{0 \leq t \leq 1}|y(t)|+\max _{0 \leq t \leq 1}\left|D_{q} y(t)\right| \\
& \leq\left(\kappa_{1}|a|^{\sigma_{1}}+\kappa_{2}|a|^{\sigma_{2}}\right)(\tau+N)+\tau+Q \\
& \leq \frac{a}{2}+\frac{a}{4}+\frac{a}{4}=a .
\end{aligned}
$$

Therefore, $T: \overline{P_{a}} \rightarrow \overline{P_{a}}$. By Lemma 3.1, $T: \overline{P_{a}} \rightarrow \overline{P_{a}}$ is completely continuous. According to Schauder's fixed point theorem, problem (1.1) has one solution.

In the following theorem, we need to note that

$$
\begin{aligned}
& \hat{f}(t, u, v)= \begin{cases}f(t, u, v), & (t, u, v) \in[0,1] \times[0, b] \times(-\infty, \infty) ; \\
f(t, b, v), & (t, u, v) \in[0,1] \times(b,+\infty) \times(-\infty, \infty),\end{cases} \\
& f^{*}(t, u, v)= \begin{cases}\hat{f}(t, u, v), & (t, u, v) \in[0,1] \times[0,+\infty) \times[-L, L] ; \\
\hat{f}(t, u,-L), & (t, u, v) \in[0,1] \times[0,+\infty) \times(-\infty,-L]) ; \\
\hat{f}(t, u, L), & (t, u, v) \in[0,1] \times[0,+\infty) \times[L,+\infty) .\end{cases}
\end{aligned}
$$


Then $f^{*} \in C\left([0,1] \times\left[0,+\infty \mid \times \mathbb{R}, \mathbb{R}^{+}\right)\right)$. Define

$$
(T y)(t)=\int_{0}^{1} G(t, q s) f^{*}\left(s, y(s), D_{q} u(s)\right) d_{q} s
$$

Theorem 3.2 Suppose $f$ is continuous on $[0,1] \times[0,+\infty) \times \mathbb{R}$ and satisfies $\left(\mathrm{H}_{1}\right)-\left(\mathrm{H}_{3}\right)$. Then problem (1.1) has at least one positive solution $y(t)$ satisfying

$$
c<\alpha(y)<b, \quad\left|D_{q} y(t)\right|<L
$$

Proof Let $\Omega_{1}=\left\{y \in X:|y(t)|<c,\left|D_{q} y(t)\right|<L\right\}, \Omega_{2}=\left\{y \in X:|y(t)|<b,\left|D_{q} y(t)\right|<L\right\}$ and $D_{1}=\{y \in X: \alpha(y)=c\}, D_{2}=\{y \in X: \alpha(y)=b\}$. By Lemma 3.1, we have proved $T: P \rightarrow P$ is completely continuous. Furthermore, we will show $\left(C_{1}\right)-\left(C_{3}\right)$ of Lemma 2.4 .

At first, by means of $\left(\mathrm{H}_{1}\right)$ and $y \in D_{1} \cap P$, we obtain

$$
\alpha(T y)=\max _{0 \leq t \leq 1}\left|\int_{0}^{1} G(t, q s) f^{*}\left(s, y(s), D_{q} u(s)\right) d_{q} s\right| \leq \frac{c}{N} \int_{0}^{1} G(1, q s) d_{q} s=c
$$

by means of $\left(\mathrm{H}_{2}\right)$ and $y \in D_{1} \cap P$, we obtain

$$
\begin{aligned}
\alpha(T y) & =\max _{0 \leq t \leq 1}\left|\int_{0}^{1} G(t, q s) f^{*}\left(s, y(s), D_{q} u(s)\right) d_{q} s\right| \\
& >\max _{0 \leq t \leq 1}\left|\int_{\frac{1}{4}}^{\frac{3}{4}} G(t, q s) \frac{b}{R} d_{q} s\right| \\
& \geq\left(\frac{1}{4}\right)^{\alpha-1} \int_{\frac{1}{4}}^{\frac{3}{4}} G(1, q s) \frac{b}{R} d_{q} s \\
& \geq \frac{1}{16} \int_{\frac{1}{4}}^{\frac{3}{4}} G(1, q s) \frac{b}{R} d_{q} s=b .
\end{aligned}
$$

Secondly, by means of $\left(\mathrm{H}_{3}\right)$ and $y \in \Omega_{2} \cap P$, we obtain

$$
\begin{aligned}
\beta(T y)= & \max _{0 \leq t \leq 1} \mid \int_{0}^{1} \frac{(1-q s)^{(\alpha-2)} t^{\alpha-2}}{\Gamma_{q}(\alpha-1)} f^{*}\left(s, y(s), D_{q} y(s)\right) d_{q} s \\
& -\int_{0}^{t} \frac{(t-q s)^{(\alpha-2)}}{\Gamma_{q}(\alpha-1)} f^{*}\left(s, y(s), D_{q} y(s)\right) d_{q} s \mid \\
< & 2 \int_{0}^{1} \frac{(1-q s)^{(\alpha-2)}}{\Gamma_{q}(\alpha-1)} \frac{L}{W} d_{q} s=L .
\end{aligned}
$$

Finally, it is easy to see that there exists a nonnegative function $p \in\left(\Omega_{2} \cap P\right) \backslash\{0\}$ such that $\alpha(y+\lambda p) \geq \alpha(y)$ for any $y \in P$ and $\lambda \geq 0$.

As a result, by Lemma 2.4 , we find that $T$ has a fixed point $y$ in $\left(\Omega_{2} \backslash \bar{\Omega}_{1}\right) \cap P$, that is, problem (1.1) has at least one positive solution $y(t)$ satisfying

$$
c<\alpha(y)<b, \quad\left|D_{q} y(t)\right|<L .
$$




\section{Example}

Example 4.1 Consider the following boundary value problem:

$$
\left\{\begin{array}{l}
-\left(D_{\frac{1}{4}}^{\frac{5}{2}} y\right)(t)=t^{2}+\left(y(t)+D_{\frac{1}{4}} y(t)\right)^{\frac{1}{2}}, \quad 0<t<1, \\
y(0)=\left(D_{\frac{1}{4}} y\right)(0)=\left(D_{\frac{1}{4}} y\right)(1)=0 .
\end{array}\right.
$$

By Theorem 3.1, it is easy to see that problem (4.1) has one positive solution.

Example 4.2 Consider the following boundary value problem:

$$
\left\{\begin{array}{l}
-\left(D_{\frac{1}{4}}^{\frac{5}{2}} y\right)(t)=\sin t+y(t)+\left(D_{\frac{1}{4}} y(t)\right)^{\frac{1}{2}}, \quad 0<t<1, \\
y(0)=\left(D_{\frac{1}{4}} y\right)(0)=\left(D_{\frac{1}{4}} y\right)(1)=0 .
\end{array}\right.
$$

Let $f\left(t, y(t), D_{\frac{1}{4}} y(t)\right)=\sin t+y(t)+\left(D_{\frac{1}{4}} y(t)\right)^{\frac{1}{2}}$. If $f\left(t, y(t), D_{\frac{1}{4}} y(t)\right)$ satisfies the following conditions:

$\left(\mathrm{H}_{1}\right) f\left(t, y(t), D_{\frac{1}{4}} y(t)\right)<1 / 4 N$ for $\left(t, y(t), D_{\frac{1}{4}} y(t)\right) \in[0,1] \times\left[0, \frac{1}{4}\right] \times\left[-10^{3}, 10^{3}\right]$;

$\left(\mathrm{H}_{2}\right) f\left(t, y(t), D_{\frac{1}{4}} y(t)\right) \geq 160 / R$ for $\left(t, y(t), D_{\frac{1}{4}} y(t)\right) \in[0,1] \times[10,160] \times\left[-10^{3}, 10^{3}\right]$;

$\left(\mathrm{H}_{3}\right) f\left(t, y(t), D_{\frac{1}{4}} y(t)\right)<10^{3} / W$ for $\left(t, y(t), D_{\frac{1}{4}} y(t)\right) \in[0,1] \times[0,160] \times\left[-10^{3}, 10^{3}\right]$, where

$$
\begin{aligned}
& G\left(1, \frac{1}{4} s\right)=\frac{\left(1-\frac{1}{4} s\right)^{\left(\frac{1}{2}\right)}-\left(1-\frac{1}{4} s\right)^{\left(\frac{3}{2}\right)}}{\Gamma_{\frac{1}{4}}\left(\frac{5}{2}\right)}, \quad N=\int_{0}^{1} G\left(1, \frac{1}{4} s\right) d_{\frac{1}{4}} s, \\
& R=\frac{1}{16} \int_{\frac{1}{4}}^{\frac{3}{4}} G\left(1, \frac{1}{4} s\right) d_{\frac{1}{4}} s, \quad W=2 \int_{0}^{1} \frac{\left(1-\frac{1}{4} s\right)^{(\alpha-2)}}{\Gamma_{\frac{1}{4}}(\alpha-1)} d_{\frac{1}{4}} s,
\end{aligned}
$$

then problem (4.2) has one positive solutions $y(t)$ satisfying

$$
\frac{1}{4}<\alpha(y)<160, \quad\left|D_{q} y(t)\right|<10^{3}
$$

by Theorem 3.2.

\section{Acknowledgements}

The authors are very grateful to the reviewers for their valuable suggestions and useful comments, which led to an improvement of this paper. The research was supported by a grant from of the National Natural Science Foundation of China (No. 11271235), the Foundation of Shanxi Datong University (2014Q10) and "131" talent project at Shanxi province (221).

Competing interests

The authors declare that they have no competing interests.

Authors' contributions

All four authors read and approved the final manuscript.

\section{Publisher's Note}

Springer Nature remains neutral with regard to jurisdictional claims in published maps and institutional affiliations. 


\section{References}

1. Jackson, F.H.: On q-functions and a certain difference operator. Trans. R. Soc. Edinb. 46, 253-281 (1908)

2. Guo, D.J., Lakshmikantham, V.: Nonlinear Problems in Abstract Cones. Academic Press, Boston (1988)

3. Al-Salam, W.A.: Some fractional $q$-integrals and q-derivatives. Proc. Edinb. Math. Soc. 15(2), 135-140 (1966/1967)

4. Bohner, M., Chieochan, R.: Floquet theory for q-difference equations. Sarajevo J. Math. 21 (8), 355-366 (2012)

5. Tariboon, J., Ntouyas, S.K., Agarwal, P.: New concepts of fractional quantum calculus and applications to impulsive fractional q-difference equations. Adv. Differ. Equ. 2015, 18 (2015)

6. Ahmad, B., Ntouyas, S.K., Tariboon, J., Alsaedi, A., Alsulami, H.H.: Impulsive fractional q-integro-difference equations with separated boundary conditions. Appl. Math. Comput. 281, 199-213 (2016)

7. Ferreira, R.A.C.: Positive solutions for a class of boundary value problems with fractional $q$-differences. Appl. Math. Comput. 61, 367-373 (2011)

8. Ferreira, R.A.C.: Nontrivial solutions for fractional $q$-difference boundary value problems. Electron. J. Qual. Theory Differ. Equ. 2010, 70 (2010)

9. Liang, S., Zhang, J.H.: Existence and uniqueness of positive solutions for three-point boundary value problem with fractional q-differences. J. Appl. Math. Comput. 40, 277-288 (2012)

10. Ahmad, B., Ntouyas, S.K., Purnaras, I.K.: Existence results for nonlocal boundary value problems of nonlinear fractional q-difference equations. Adv. Differ. Equ. 2012, 140 (2012)

11. Ahmad, B., Ntouyas, S.K.: Existence of solutions for nonlinear fractional q-difference inclusions with nonlocal Robin (separated) conditions. Mediterr. J. Math. 10, 1333-1351 (2013)

12. Thiramanus, P., Tariboon, J.: Nonlinear second-order q-difference equations with three-point boundary conditions. Comput. Appl. Math. 33, 385-397 (2014)

13. Zhou, W.X., Liu, H.Z: Existence solutions for boundary value problem of nonlinear fractional $q$-difference equations. Adv. Differ. Equ. 2013, 113 (2013)

14. Yu, C.L., Wang, J.F.: Existence of solutions for nonlinear second-order q-difference equations with first-order q-derivatives. Adv. Differ. Equ. 2013, 124 (2013)

15. Zhao, Y.L., Chen, H.B., Zhang, Q.M.: Existence and multiplicity of positive solutions for nonhomogeneous boundary value problems with fractional q-derivatives. Bound. Value Probl. 2013, 103 (2013)

16. Yu, C.L., Wang, J.F.: Eigenvalue of boundary value problem for nonlinear singular third-order $q$-difference equations. Adv. Differ. Equ. 2014, 21 (2014)

17. Li, X.H., Han, Z.L., Sun, S.R., Zhao, P.: Existence of solutions for fractional q-difference equation with mixed nonlinear boundary conditions. Adv. Differ. Equ. 2014, 326 (2014)

18. Zhao, Q.B., Yang, W.G.: Positive solutions for singular coupled integral boundary value problems of nonlinear higher-order fractional q-difference equations. Adv. Differ. Equ. 2015, 290 (2015)

19. Jiang, M., Zhong, S.M.: Existence of extremal solutions for a nonlinear fractional $q$-difference system. Mediterr. J. Math. 13, 279-299 (2016)

20. Zhai, C.B., Ren, J.: Positive and negative solutions of a boundary value problem for a fractional q-difference equation. Adv. Differ. Equ. 2017, 82 (2017)

21. Sitthiwirattham, T:: On a fractional $q$-integral boundary value problems for fractional $q$-difference equations and fractional $q$-integrodifference equations involving different numbers of order $q$. Bound. Value Probl. 2016, 12 (2016)

22. Patanarapeelert, N., Sriphanomwan, U., Sitthiwirattham, T.: On a class of sequential fractional $q$-integrodifference boundary value problems involving different numbers of $q$ in derivatives and integrals. Adv. Differ. Equ. 2016, 148 (2016)

23. Kac, V., Cheung, P.: Quantum Calculus. Springer, New York (2002)

24. Agarwal, R.P.: Certain fractional $q$-integrals and $q$-derivatives. Proc. Camb. Philos. Soc. 66, 365-370 (1969)

25. Rajković, P.M., Marinković, S.D., Stanković, M.S.: Fractional integrals and derivatives in q-calculus. Appl. Anal. Discrete Math. 1(1), 311-323 (2007)

26. Ferreira, R.A.C.: Nontrivial solutions for fractional q-difference boundary value problems. Electron. J. Qual. Theory Differ. Equ. 70, 10 (2010)

27. Guo, Y., Ge, W.: Positive solutions for three-point boundary value problems with dependence on the first order derivative. J. Math. Anal. Appl. 290, 291-301 (2004)

\section{Submit your manuscript to a SpringerOpen ${ }^{\circ}$ journal and benefit from:}

- Convenient online submission

- Rigorous peer review

- Open access: articles freely available online

- High visibility within the field

Retaining the copyright to your article

Submit your next manuscript at $\boldsymbol{~ s p r i n g e r o p e n . c o m ~}$ 